Ann. Génét. Sél. anim., I978, 10 (3), 39I-40r.

\title{
Etude du croisement entre les races ovines Fleischschaf et Aragonaise pour les caractères de croissance et d'abattage
}

\author{
M. VALLS ORTIZ, M. ESPEJO ('), B. BONAITI (2) \\ Departamento de Produccion Animal Pastos y Forrajes \\ CRIDA o3 I.N.I.A. \\ Apdo 202, Zaragoza, Espagne
}

\begin{abstract}
Résumé
Ce travail concerne l'étude du croisement réciproque entre les races ovines Fleischschaf et Aragonaise. Chacune des deux races est représentée par I 70 brebis et 6 béliers. Le schéma permet d'étuđier l'influence des effets directs, maternels et d'hétérosis sur la croissance des agneaux jusqu'à l'âge de 75 jours. Pour les résultats d'abattage, seules sont obtenues les données des deux croisements réciproques et de la race pure Aragonaise.

Cette expérience met en évidence le grand intérêt du croisement industriel Fleischschaf sur les brebis Avagonaises qui entraîne une augmentation de la vitesse de croissance et une réduction de l'adiposité des carcasses.
\end{abstract}

\section{Introduction}

Le croisement industriel ovin est de plus en plus répandu dans la vallée de 1'Ebre (Esspagne). L'administration contribua à ce développement par l'implantation de la race Fleischschaf qui, tout en étant de type Mérinos, apportait une bonne conformation et de bonnes capacités d'adaptation.

Plusieurs essais ont permis de comparer certaines races, dont le Fleischschaf, pour le croisement industriel sur les races locales (Espejo et VALLS ORTIZ, I976). On a pu mettre en évidence (Sierra, ig69; Espejo, Valis Ortiz et Colomer, I974) les avantages de la race Fleischschaf dans les conditions d'engraissement et de commercialisation des carcasses de la région de Saragosse.

La recherche d'une plus grande intensification de la production pourrait conduire à utiliser comme mères les femelles issues d'un simple croisement lors-

(1) Departamento de Produccion Animal - CRIDA.08 - INIA, Apdo. 22. BADAJOZ, Espagne.

$\left({ }^{2}\right)$ Station de Génétique quantitative et appliquée, Centre national de Recherches zootechniques, I.N.R.A., 78350 Jouy-en-Josas, France. 
qu'un niveau d'alimentation satisfaisant est possible. Dans le but d'étudier cette possibilité qui intéresse déjà quelques éleveurs, un schéma d'accouplements réciproques fut mis en place au cours de la campagne I972-I973 entre les races Fleischschaf et Aragonaise ( $)$ pour permettre une estimation des effets directs, maternels et d'hétérosis sur les caractères de croissance et de reproduction. Nous présentons ici les résultats concernant les caractères de croissance et de qualité des carcasses.

\section{I. - Matériel et méthodes}

Les animaux de la race Fleischschat utilisés dans notre comparaison proviennent d'un troupeau qui, depuis sa création en I964 par l'importation de 50 agnelles et de 2 béliers, n'a pas subi de sélection importante pour pouvoir satisfaire aux besoins de multiplication. Ce troupeau semble être très bien adapté aux conditions locales. La race Aragonaise, comparée à la Fleischschaf est d'un format très inférieur (45 kg contre $70 \mathrm{~kg}$ pour les femelles adultes). Le schéma d'accouplement réciproque met en œuvre I7o brebis et 6 béliers par race; mais à cause de certaines contraintes techniques, trois béliers sont utilisés en race pure et les trois autres en croisement. Les effectifs des agneaux nés en avril I973 de ces accouplements sont donnés dans le tableau $\mathrm{I}$.

Les brebis Aragonaise reçoivent habituellement $300 \mathrm{~g}$ de céréales et $\mathrm{I} \mathrm{kg}$ de luzerne déshydratée. Cependant pendant la période d'allaitement, la quantité de céréales distribuée est de I $\mathrm{kg}$. Les brebis Fleischschaf reçoivent une ration supérieure de $20 \mathrm{p}$. Ioo. La lutte est réalisée durant le mois de novembre avec trois béliers par lot. Les agneaux restent avec leur mère et disposent à partir de 1'âge de 20 jours, d'un concentré commercial ( 17 p. roo de protéines brutes) qui est progressivement remplacé pendant la période d'engraissement par un mélange de céréales (maïs et orge). La durée d'accès aux concentrés limitée à 6 heures durant la journée peut réduire le niveau d'ingestion des agneaux. Après le sevrage réalisé vers 80 jours ( \pm 5 jours), les agneaux ne reçoivent plus que le mélange de céréales.

Les agneaux sont pesés à la naissance, puis tous les 15 jours. Des poids à âge-type sont calculés par intrapolation linéaire à Io, 30, 60 et 75 jours. Des gains moyens quotidiens sont ensuite calculés entre ro et 30 jours d'une part et 60 et 75 jours d'autre part. Ces périodes d'observation de la croissance sont choisies de telle sorte qu'elles représentent au mieux respectivement la production laitière de la mère et le potentiel de croissance des agneaux. Après le sevrage et seulement pour les 43 agneaux mâles contrôlés en carcasse, une pesée hebdomadaire permet de réaliser un poids d'abattage le plus près possible de $32 \mathrm{~kg}$ (ceci correspond à un âge moyen d'abattage de 132 jours). Après 20 heures de ressuyage, les carcasses sont pesées, mesurées et découpées selon la méthodologie déjà décrite par ColOMER et EsPEJo (I972). Le rendement est estimé par le rapport du poids de carcasse froide sur le poids vif à l'abattoir. Les observations réalisées sur la carcasse sont les suivantes : longueur de la carcasse $(L)$, épaisseur de cuisse $(G)$, longueur jarretsymphyse $(\mathrm{F})$, épaisseur de gras sur la $\mathrm{I}_{3}{ }^{\mathrm{e}}$ côte $(\mathrm{C})$, poids du gras périrénal, surface de la $I 3^{e}$ côte et poids de l'os canon.

Les données sont analysées selon un modèle de moindres carrés tenant compte des effectifs déséquilibrés. Le modèle à effets fixés suivant a été utilisé :

(1) Rasa Aragonesa, en espagnol. 
$\mathrm{Y}_{i j k l}=\mathrm{M}_{i}+\mathrm{S}_{j}+\mathrm{G}_{k}+(\mathrm{M} . \mathrm{S})_{i j}+(\mathrm{M} . \mathrm{G})_{i k}+(\mathrm{S} . \mathrm{G})_{j k}+e_{i j k l}$.

$\mathbf{M}_{i}=$ effet fixé du mode de naissance $(i=\mathbf{I}$ et 2$)$.

$\mathrm{S}_{j}=$ effet fixé du sexe $(j=\mathrm{I}$ et 2$)$.

$\mathrm{G}_{k}=$ effet fixé du type génétique $(k=\mathrm{I}$ à 4$)$.

$(\mathrm{M} . \mathrm{S})_{i j},(\mathrm{M} . \mathrm{G})_{i k}$ et $(\mathrm{S} . \mathrm{G})_{j k}$ les 3 interactions entre les 3 facteurs.

$e_{i j k l}=$ effet résiduel aléatoire.

Pour les données d'abattage des agneaux mâles, il n'est évidemment pas tenu compte dans le modèle de l'effet sexe et des interactions correspondantes. Sauf pour l'analyse du rendement, le poids de carcasse est introduit dans le modèle comme covariable.

Les estimations des effets directs, maternels et d'hétérosis sont obtenues à partir des estimées correspondant aux quatre types génétiques. Les écarts-types sont calculés avec 1'inverse de la matrice des effectifs et les écarts-types résiduels. Les tests de signification de ces effets sont ensuite réalisés selon la méthode de test de Student. Puisque les agneaux Fleischschaf ne sont pas abattus, seul l'effet maternel est estimé pour les caractères d'abattage.

\section{II. - Résultats}

\section{a. - Critères de croissance}

\section{TABLEAU I}

Effets $d u$ mode de naissance, du sexe et du génotype de l'agneau sur les caractèves de croissance

Effects of litter size, sex and lamb's genotype on the growth

\begin{tabular}{|c|c|c|c|c|c|c|c|c|}
\hline & & \multirow{2}{*}{ Effectif } & \multicolumn{4}{|c|}{ Poids (kg) } & \multicolumn{2}{|c|}{ Croissance $(\mathrm{g} / \mathrm{j})$} \\
\hline & & & $\begin{array}{l}\text { Nais- } \\
\text { sance }\end{array}$ & Io jours & 30 jours & 75 jours & $\begin{array}{c}10 \text { à } \\
30 \text { jours }\end{array}$ & $\begin{array}{c}60 \text { à } \\
75 \text { jours }\end{array}$ \\
\hline \multicolumn{9}{|l|}{ Mode de naissance : } \\
\hline Test. . . . & : & $140-95$ & $\begin{array}{l}0,02 \\
* *\end{array}$ & ** & $\begin{array}{l}1,7 \\
* *\end{array}$ & 2,1 & $\begin{array}{l}34 \\
* *\end{array}$ & $\begin{array}{l}\text { I0 } \\
\text { NS }\end{array}$ \\
\hline Sexe : & & & & & & & & \\
\hline $\begin{array}{l}\text { Mâle-femelle . . } \\
\text { Test . . . . . }\end{array}$ & $\dot{.}$ & I $27-108$ & $\begin{array}{c}0,36 \\
* *\end{array}$ & $\begin{array}{l}0,4 \\
* *\end{array}$ & $\begin{array}{l}0,7 \\
* *\end{array}$ & $\begin{array}{l}1,9 \\
* *\end{array}$ & $\begin{array}{l}16 \\
*\end{array}$ & $\underset{* *}{36}$ \\
\hline \multicolumn{9}{|l|}{ Génotype $\left({ }^{1}\right)$ : } \\
\hline Fleischschaf & . & 49 & 3,88 & $6, \mathrm{I}$ & 10,6 & 21,2 & 227 & 248 \\
\hline Arag. $\times$ Fleisch & . . & $3^{8}$ & 3,62 & 5,9 & $10, \mathrm{I}$ & 19,7 & 213 & 226 \\
\hline Fleisch. $\times$ Arag. & . . & $8 \mathrm{r}$ & 3,35 & 5,4 & 9,7 & 19,5 & $2 \mathrm{II}$ & 228 \\
\hline Aragonais . . & . . & 67 & 2,95 & 4,8 & 8,5 & I6,7 & I 84 & I 82 \\
\hline \multirow{3}{*}{$\begin{array}{l}\text { Test } \\
\text { Moyenne } \\
\text { Écart-type résiduel }\end{array}$} & & & $* *$ & $* *$ & $* *$ & $* *$ & *** & $* *$ \\
\hline & . . . & 235 & 3,45 & 5,5 & 9,7 & 19,3 & 209 & $22 \mathrm{I}$ \\
\hline & & & 0,65 & 0,9 & $I, 8$ & 3,5 & 49 & $7 \mathrm{I}$ \\
\hline
\end{tabular}

** Significatif au seuil de I p. roo.

* Significatif au seuil de 5 p. roo.

(1) Pour les génotypes croisés, la race du père est donnée en premier et celle de la mère en second. 
Les effets du sexe et du mode de naissance sont significatifs pour tous les critères de poids (tabl. I). Mais les effets du mode de naissance, proportionnellement plus importants au début, diminuent, tandis que ceux du sexe augmentent, avec 1'âge. Ceci suggère que l'extériorisation des différences de potentiel de croissance s'amplifie avec l'âge. Ceci est encore plus significatif pour les deux vitesses de croissance.

Le génotype a un effet significatif sur tous les critères. La décomposition de l'effet " type génétique " en effets directs, maternels et d'hétérosis (tabl. 2) fait apparaître trois choses. Les effets directs de la race Fleischschaf sont supérieurs de I6 à 36 p. roo à ceux de la race Aragonaise et cette différence augmente avec l'âge. Les effets maternels sont aussi à l'avantage du Fleischschaf. Ils sont significatifs à la naissance, diminuent et disparaissent après l'âge de 30 jours. Au contraire, l'effet d'hétérosis, inférieur aux effets maternels pour les premiers mois, devient plus important après 45 jours tout en restant non significatif.

\section{TABLEAU 2}

Estimées des effets directs, maternels et d'hétérosis sur les variables de croissance Estimation of direct, maternal and heterosis effects on growth

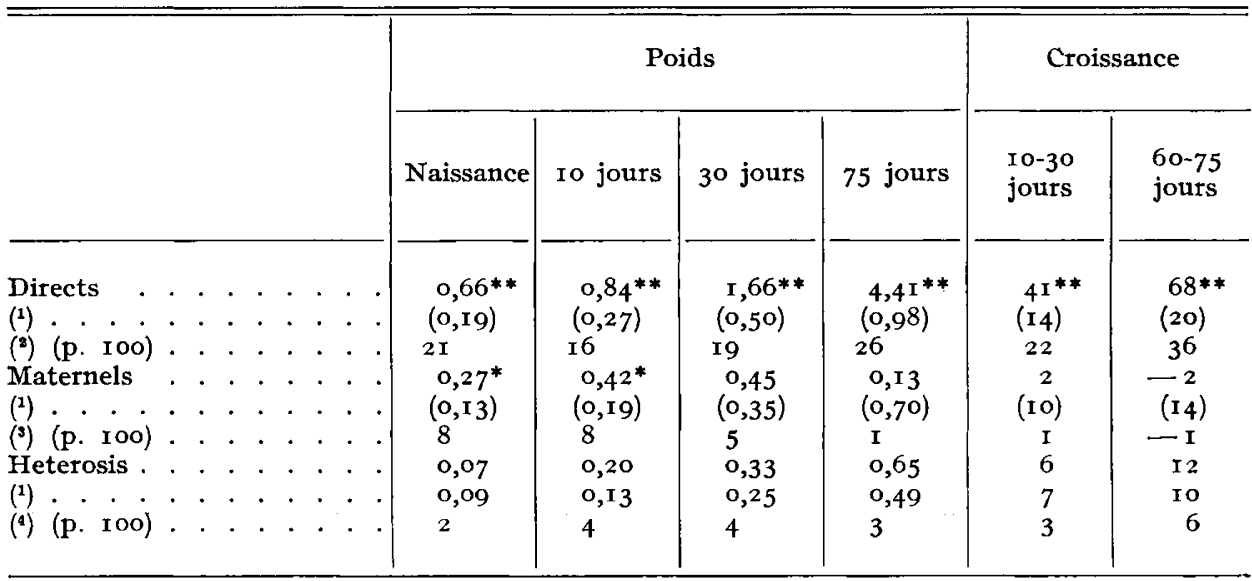

(1) Écart-type de l'estimée

(2) $[(\mathbf{F}+\mathbf{F A})-(\mathbf{A}+\mathbf{A F})] /(\mathbf{A}+\mathbf{A F})$.

(3) $(\mathrm{AF}-\mathrm{FA}) / \mathrm{FA}$.

(A : Aragonais; F : Fleischschaf).

(4) $[(\mathbf{A F}+\mathbf{F A})-(\mathbf{F}+\mathbf{A})] /(\mathbf{F}+\mathbf{A})$.

** Significatif au seuil de I p. Ioo.

* Significatif au seuil de 5 p. Ioo.

\section{b. - Caractères d'abattage}

L'absence de différences significatives, selon le génotype ou le mode de naissance, pour le poids d'abattage mesuré en ferme $(32,5 \mathrm{~kg} \pm \mathrm{I}, 2)$ ou à l'abattoir (30 $\mathrm{kg} \pm \mathrm{I}, 2)$ montre que le schéma expérimental a bien été respecté. Les effets observés sur le poids de carcasse, qui est utilisé comme covariable dans le modèle d'analyse des autres variables, sont faibles et non significatifs. 
CROISEMENTS " FLEISHSCHAF XARAGONAISE "

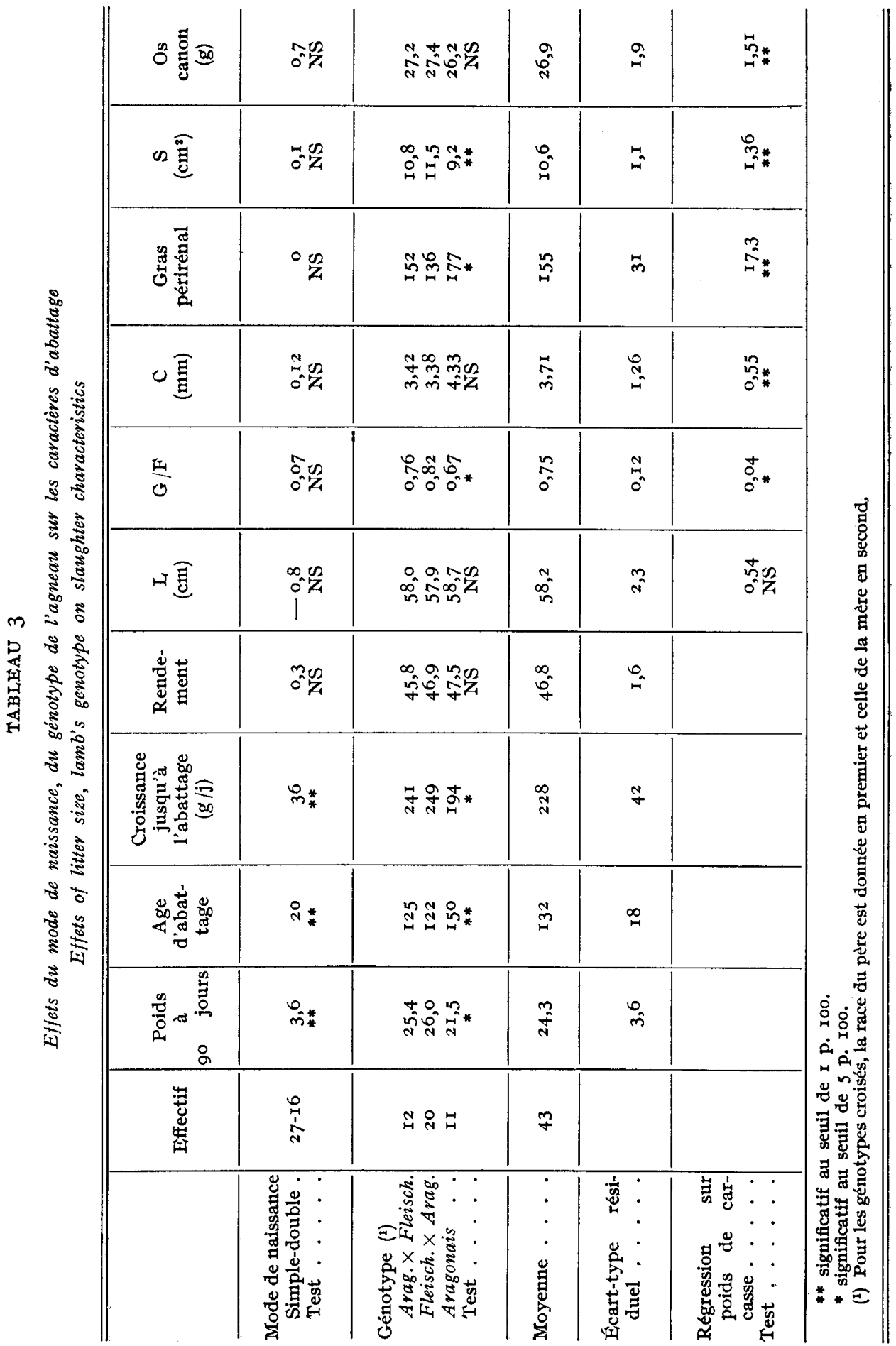


Les résultats présentés dans le tableau 3 montrent que le mode de naissance n'a pas d'effet significatif sur les variables. Aucune variable ne présente une interaction significative entre mode de naissance et génotype. Il existe par contre d'importantes différences entre génotypes. Le rendement des agneaux Aragonais est légèrement inférieur à celui des Fleischschaf $\times$ Aragonais et ce dernier à celui des Aragonais $\times$ Fleischschaf. La conformation est améliorée par le croisement, particulièrement pour la variable $\mathrm{G} / \mathrm{F}$ (rapport de l'épaisseur de cuisse à la longueur jarret-symphyse), seule variable présentant un effet significatif. L'état d'engraissement est inférieur chez les croisés et la différence n'est significative que pour le gras périrénal dont la variabilité résiduelle est plus faible que pour l'épaisseur de gras dorsal. La surface du muscle sur la $13^{\mathbf{e}}$ côte est supérieure chez les croisés qui diffèrent aussi entre eux selon la race de mère. Le poids d'os canon est plus élevé chez les croisés mais cette différence n'est pas significative. Les effets observés sur le rendement lors de la découpe sont tous faibles et non significatifs (tab1. 4).

TABLEAU 4

Effets du mode de naissance,

du génotype de l'agneau sur les résultats de découpe des carcasses

Effects of litter size, lamb's genotype on the carcass cutting results

\begin{tabular}{|c|c|c|c|c|c|c|c|}
\hline & Gigot & $\begin{array}{l}\text { Côtes } \\
+ \\
\text { filet }\end{array}$ & $\begin{array}{l}\text { Carré } \\
\text { décou- } \\
\text { vert }\end{array}$ & Epaule & Collier & Poitrine & Queue \\
\hline $\begin{array}{c}\text { Mode de naissance : } \\
\text { Simple-double } \\
\text { Test . . . . . }\end{array}$ & $\begin{array}{c}-0,47 \\
\mathrm{NS}\end{array}$ & $\begin{array}{l}\text { 0,09 } \\
\text { NS }\end{array}$ & $\begin{array}{c}-0,08 \\
\text { NS }\end{array}$ & $\begin{array}{c}-0,32 \\
\mathrm{NS}\end{array}$ & 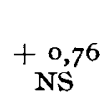 & $\mathrm{NS}^{0,42}$ & $\begin{array}{c}-0,45 \\
\text { NS }\end{array}$ \\
\hline $\begin{array}{l}\text { Génotype }\left({ }^{1}\right): \\
\text { Arag. } \times \text { Fleisch. } \\
\text { Fleisch. } \times \text { Arag. } \\
\text { Aragonais . . . } \\
\text { Test . . . . . . }\end{array}$ & $\begin{array}{l}29,50 \\
30,24 \\
3^{\circ}, 26 \\
\text { NS }\end{array}$ & $\begin{array}{l}22,23 \\
22,36 \\
22,61 \\
\text { NS }\end{array}$ & $\begin{array}{l}7,94 \\
7,63 \\
7,83 \\
\text { NS }\end{array}$ & $\begin{array}{l}19,03 \\
\text { I } 8,34 \\
\text { I } 7,81 \\
\text { NS }\end{array}$ & $\begin{array}{l}6,56 \\
6,99 \\
6,25 \\
\text { NS }\end{array}$ & $\begin{array}{l}13,08 \\
12,62 \\
14,21 \\
\text { NS }\end{array}$ & $\begin{array}{l}\mathbf{I}, 77 \\
\mathbf{I}, 60 \\
\mathrm{I}, \mathbf{1 3} \\
\mathrm{NS}\end{array}$ \\
\hline 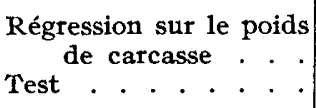 & $-\underset{* *}{0,62}$ & $\begin{array}{l}\text { o,36 } \\
\text { NS }\end{array}$ & $\begin{array}{c}-\mathrm{o}, 32 \\
\mathrm{NS}\end{array}$ & $\begin{array}{c}-0,02 \\
\mathrm{NS}\end{array}$ & $\begin{array}{l}\text { o,36 } \\
\mathrm{NS}\end{array}$ & $\begin{array}{c}-0,005 \\
\text { NS }\end{array}$ & $\stackrel{0,24}{N S}$ \\
\hline Moyenne (p. Ioo) & 30,0 & 22,4 & 7,8 & I 8,5 & 6,6 & 13,3 & $\mathrm{I}, 5$ \\
\hline Écart-type résiduel & $\mathrm{I}, 02$ & 1,5 & $\mathrm{I}, \mathrm{O}$ & $\mathrm{I}, 2$ & 1,3 & $\mathrm{I}, 9$ & 0,6 \\
\hline
\end{tabular}

** Significatif au seuil de I p. roo.

* Significatif au seuil de 5 p. roo.

(1) Pour les génotypes croisés, la race du père est donnée en premier et celle de la mère en second. 


\section{III. - Discussion}

Les moyennes de tous les critères de croissance apparaissent très faibles en comparaison des résultats obtenus avec les mêmes races (EsPEJO et VALIS ORTIZ, I976). Ceci peut s'expliquer par les conditions générales d'alimentation des brebis et des agneaux et en particulier peut-être par la limitation de la durée d'accès des agneaux aux concentrés. Le régime de fin d'engraissement (maïs + orge) est par contre favorable à la formation des dépôts adipeux.

L'évolution avec l'âge des différents facteurs de variation semble relativement homogène. D'une part, l'importance des facteurs d'influence maternelle (mode de naissance et effets maternels au sens strict) diminue de la naissance au sevrage tandis que celles des facteurs génétiques directs (sexe, génotype de l'agneau, hétérosis) augmente avec l'âge.

Dans le jeune âge l'influence maternelle est déterminante car le lait mäternel constitue la seule source d'alimentation. Ensuite l'uniformisation des conditions d'alimentation par l'apport de concentrés permet aux effets génétiques directs de s'exprimer. Les faibles valeurs d'hétérosis observées peuvent s'expliquer par un faible niveau de croissance des agneaux. PETERS et HEANEY (I974) ont déjà observé que les effets d'hétérosis sur la vitesse de croissance sont plus importants lorsque les agneaux sont élevés artificiellement et donc sans limitation alimentaire.

La forte variation avec l'âge des effets maternels appelle aussi quelques remarques. Cette évolution a déjà été observée par HoLTMANN et BERNARD (I969) et par WiENER et HAYTER (I975). Mais il semble que dans notre cas l'in fluence maternelle utérine, peut-être liée au format maternel, soit beaucoup plus importante que l'in fluence postnatale qui, elle, est liée à la production laitière. Nos observations indiqueraient peut-être une certaine faiblesse relative de la race Fleischschaf pour la production laitière. DrCKERSON et al. (I972) ont déjà rapporté des résultats indiquant une opposition entre des effets maternels importants avant la naissance et faibles après la naissance. Mais il ne faudrait pas généraliser notre résultat d'une part parce que les conditions de milieu ont pu empêcher l'extériorisation du potentiel laitier du Fleischschaf et d'autre part parce que ce dispositif expérimental ne fait intervenir que 2 génotypes pour effectuer cette évaluation.

Les différences observées entre génotypes pour l'état d'engraissement doivent être rapprochées du fait que le même poids d'abattage était prévu pour des génotypes qui diffèrent très largement par leur poids adulte. Elles reflètent donc plus des différences de degré de maturité à l'abattage. Par contre on ne relève pas de différences apparentes dans la distribution des dépôts adipeux entre la race $\mathrm{Ara}$ gonaise et les croisés Fleischschaf. La comparaison avec les Fleischschaf aurait peut-être pu mettre en évidence de telles différences mais on peut, cependant, rapprocher ce résultat de l'origine Merinos du Fleischschaf.

Une légère supériorité du Fleischschaf est observée pour la conformation de carcasse qui est sans doute plus indépendante du degré de maturité.

L'interprétation des effets maternels sur les caractères de la carcasse n'est pas évidente. L'estimation de ces effets pourrait être biaisée par des différences d'échantillonnage entre les parents mâles et femelles. Mais cette hypothèse ne semble guère plausible dans la mesure où pour chacune des deux races les reproducteurs mâles et femelles sont issus đu même troupeau. Ces effets s'observent sur le rendement et sur le gras de rognon. Pour cette dernière variable, l'effet maternel pourrait être le résultat d'un apport nutritif maternel plus important pour la brebis Fleischschaf. Mais les résultats observés sur la croissance montre que l'effet n'était favorable que durant la phase prénatale. Cette hypothèse est cepen- 
dant vraisemblable dans la mesure où le seul dépôt de gras sujet à des effets maternels est le gras de rognon qui est de développement plus précoce et dont le nombre total d'adipocytes est déterminé, chez l'agneau, à la naissance (VEZINHEr, NouguEs et VIGNERON, I976).

Lors de la découpe de la carcasse, les proportions moyennes de chaque morceau sont comparables à celles observées par CoLOMER et ESPEJO (I972) sur des agneaux croisés Manchega $\times$ Aragonais. En accord avec les observations de BocCARD et Dumont (I962), aucune différence importante n'est observée entre les trois génotypes (tabl. 4).

\section{IV. - Conclusion}

L'ensemble des résultats révèle le grand intérêt du croisement industriel Fleischschaf sur la population femelle Aragonaise (tabl. 5). Pour les caractères observés lors de l'abattage, il n'est pas possible de déterminer la part relative de l'avantage dû aux effets directs ou aux effets d'hétérosis. Ces avantages tiennent essentiellement en deux points : la réduction des coûts de production et la possibilité de produire des carcasses plus lourdes avec une composition semblable. L'augmentation du potentiel de croissance entraîne une réduction de la durée d'engraissement de l'ordre de I9 $\mathrm{p}$. I00. Si on suppose que les besoins d'entretien sont réduits d'autant, et que ceux-ci représentent la moitié des besoins totaux, on pourrait conclure à une réduction des besoins de l'ordre de Io p. Ioo. En outre, la réduction d'adiposité des carcasses doit aussi s'accompagner d'une meilleure efficacité de la transformation des aliments. Ceci laisse donc supposer une efficacité alimentaire supérieure d'au moins ro p. Ioo pour les croisés. Srmo (I976) trouvait également un avantage des croisés Ile-de-France de $20 \mathrm{p}$. Ioo pour la consommation d'aliment durant la croissance entre I5 et $30 \mathrm{~kg}$. La plus faible adiposité des carcasses des agneaux croisés permet la commercialisation de carcasses plus lourdes. L'estimation obtenue pour le coefficient de régression de l'épaisseur du gras dorsal sur le poids de carcasse permet de supposer que les agneaux croisés pourraient être abattus à un poids de carcasse supérieur de $I, 6 \mathrm{~kg}$ soit une augmentation de I2 p. Ioo. Ce dernier avantage des agneaux croisés peut, cependant ne pas être facilement valorisé dans les conditions actuelles de commercialisation en Espagne qui imposent de faibles poids d'abattage.

Rę̧u pour publication en décembre 1978 .

\section{Remerciements}

Les auteurs remercient la Diputacion Provincial de Zaragoza pour les facilités accordées pour la réalisation de cette expérience, plus particulièrement MM. PASTOR, LAZARO et CALLEN ainsi que M. LAHOZ de l'I.N.I.A. pour leur participation à la collecte des données et MM. BIBE, RicordeaU, Flamant (I.N.R.A.) pour leurs utiles critiques et suggestions à la lecture du manuscrit. 


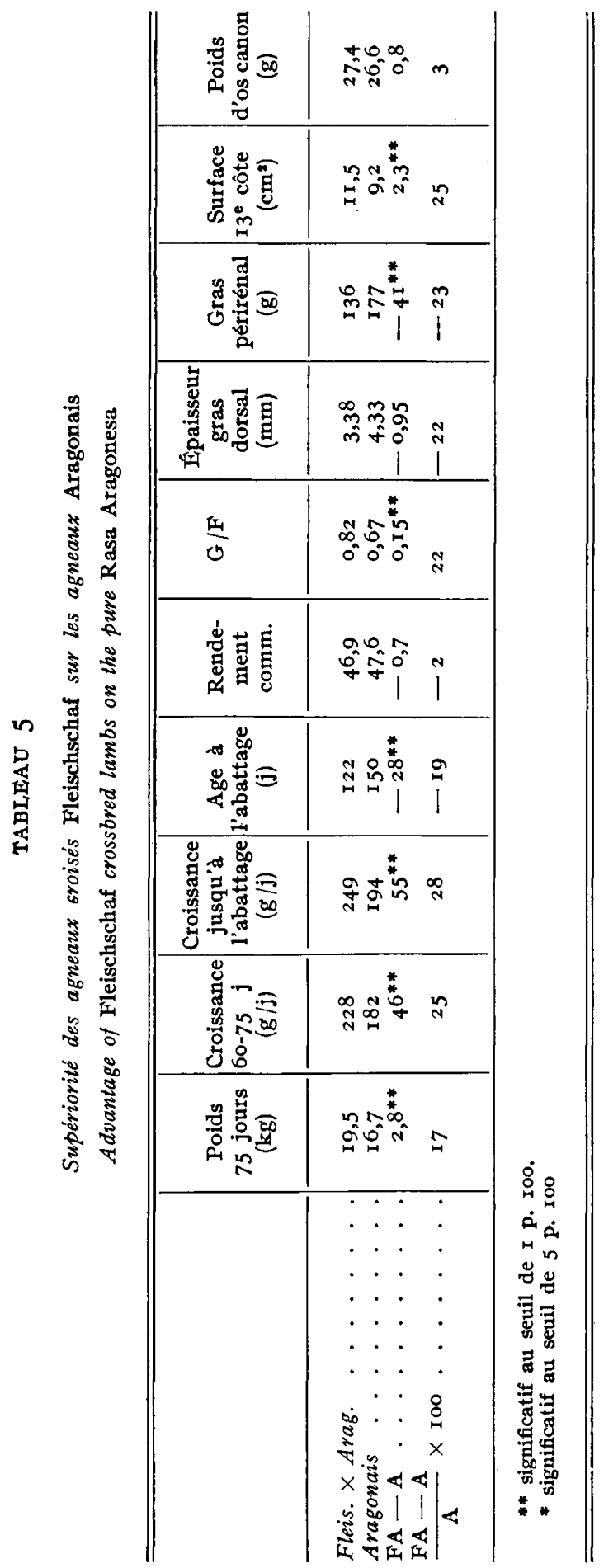




\section{Summary}

\section{Study of crossbreeding between the Fleischschaf and the Aragon sheep for the characteristics of growth and slaughtering}

This study concerns the reciprocal crossbreeding between the breeds Fleischschat and Rasa Avagonesa. Each of them is represented by $\mathrm{I} 70$ ewes and 6 rams. The experimental scheme allows the study of direct and maternals effects and the heterosis on the lamb's growth up to 75 days. But at slaughter, the data are got only for the two reciprocal crossbreds and for the Rasa Avagonesa purebreed. This experiment shows the great interest of terminal crossing on the Rasa Aragonesa with Fleischschat rams. This strategy leads to a greater growth rate and a less fatty carcass.

\section{Resumen}

Estudio del cruzamiento entre las razas ovinas Fleischschaf $y$ Aragon. Efectos sobre el crecimiento de las corderas y sobre las caracteristicas de la canal

Se estudia el crirce recíproco entre las razas Fleischschaf y la Rasa Avagonesa. Cada una de estas razas estaba' Tespresentada por I 70 hembras y 6 machos. El esquema empleado permite estudiar la influencia de los efectos directos, maternales y de heterosis sobre el crecimiento de los corderos hasta la edad de 75 dias. Los resultados de sacrificio conciernen solamente a los dos cruces recíprocos y a la raza Rasa Aragonesa.

Esta experiencia pone de manifiesto el gran interés del cruce industrial Fleischschaf sobre la raza Rasa Aragonesa. El cruce se traduce fundamentalmente en el aumento de la velocidad de crecimiento y la reduccion del estado de engrasamiento de las canales.

\section{Références bibliographiques}

BOCCARD R., DUmont B. L., I96o. Étude de la production de viande chez les ovins. II-Variation de l'importance relative des différentes régions corporelles de l'agneau de boucherie. $A n n$. Zootech., 9, 355-363.

COLOMER F., ESPEJO M., 1972. Influence du poids d'abattage et du sexe sur les performances de boucherie des agneaux issus du croisement Manchego $\times$ Rasa aragonesa. Ann. Zootech., 21, 4OI-4I4.

Dickerson G. E., Glimp H. A., Tuma H. J., Gregory K. E., I972. Genetic resources for efficient meat production on sheep. Growth and carcass characteristics of ram lambs of seven breeds. J. Anim. Sci., 34, 940-95I.

ESPEJO M., VALLS ORTIZ M., 1976. La produccion de carne ovina y el cruzamiento industrial en Espana. Bull. tech. Dép. Genet. anim. (Inst. natn. Rech. agron., Fr.), 25, 45-64.

ESPEJO M., VALLS ORTIZ M., Colomer F., 1974. Ensayo comparativo de cruce de una raza ovina Espanola con moruecos de raza Finlandesa y con otros de aptitud carnica. I-Congreso mundial de genetica aplicada a la produccion ganadera. Vol. III : 94I-949.

HolTMANN W. B., BERNARD C., I969. Effect of general combining ability and maternal ability of Oxford, Suffolk and North Country Cheviot breeds of Sheep on growth performance of lambs. J. Anim. Sci., 28, 155-r6r.

PETERS H. I., HEANEY D. P., I974. Heterosis, breed-of-sire and breed-of-dam effects on growth rates of lambs reared artificially or with their dams. Can. J. Anim. Sci., 54, I9-22.

SIERRA I., I969. Resultados del cruce industrial en ganado ovino de Rasa aragonesa. Anal. Est. Exper. Aula Dei, 9, 373-380. 
Simo L., 1976. Principales factores que afectan a las caracteristicas productivas de los corderos de raza Rasa Aragonesa $y$ su cruce con.Ile-de-France en condiciones reales de explotacion en zona arida. Tesis doctoral ETSIA, Madrid.

Vezinhet A., Nougues J., VIGNERON P., 1976. Aspects généraux du développement et de la croissance des tissus musculaires et adipeux : caractéristiques chez les ovins. Journées de la Recherche ovine et caprine. Paris 27-43 Ed. Itovic, Paris.

WIENER G., HAXTER S., r975. Maternal performance of sheep as affected by breed, crossbreeding and other factors. Anim. Prod., 20, r9-3o.

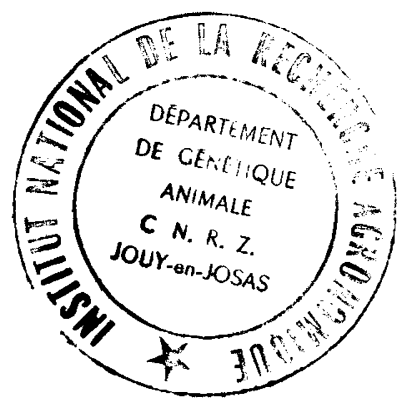

